\title{
From micelle to microemulsion: an investigation of structural changes using molecular dynamics
}

\author{
Amir Amania,b \& Milad Amanic
}

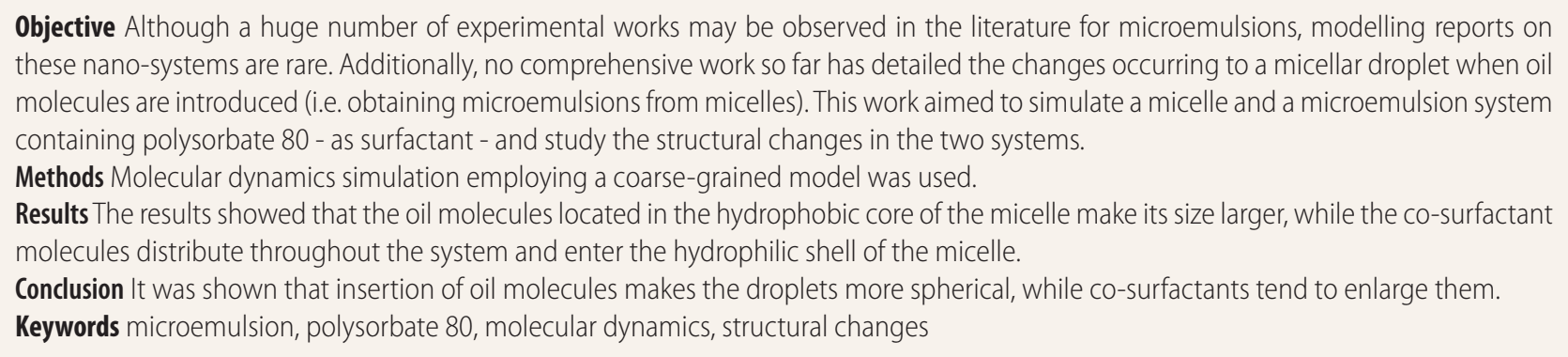

\section{Introduction}

"Microemulsion" was first defined in 1981 as "a system of water, oil, and amphiphile which is a single optically isotropic and thermodynamically stable liquid solution." ${ }^{1}$ When preparing a mixture of oil and water, in the absence of surfactant(s), a twophase system is formed. By addition of a surface active agent (surfactant), the surfactant molecules move to the interface of oil and water and lower the interfacial tension. The surfactant molecules then try to trap some oil (water) molecules within their aggregates and make a nanodispersion of oil (water) in the water (oil) phase. Addition of more surfactant molecules will eventually "solubilize" the whole oil (water) phase, in continuous phase., ${ }^{2,3}$ This forms the microemulsion particles.

Structurally, microemulsions are similar to micelle droplets (i.e. aggregates of surfactant molecules, ${ }^{4}$ while an internal phase is located within the droplet. The internal phase should be immiscible with the continuous phase. Usually, in addition to the surfactant molecules, a short chain alcohol is added to the preparation to provide flexibility of interface and promote the stability of the microemulsions. Such alcohols are called co-surfactants. ${ }^{5}$

It is a common practice to employ molecular simulation approaches in order to fill the gaps left by experimentalist and provide insight into dynamics and thermodynamic quantities at molecular levels. Considering the literature, an ocean of experiments can be found on microemulsions. Also, numerous simulation works have been reported on micelles. However, limited number of molecular simulation reports is available for microemulsions. In works by Baaden et al., the role and behavior of tri-n-butyl phosphate (TBP) as a surfactant, a modifier of interface, or a solute in a mixture of water and oil was studied. The results of this molecular dynamics (MD) simulation showed that hydrogen bonding at high TBP concentrations may lead to the formation of microemulsions. ${ }^{6,7} \mathrm{~A}$ microemulsion system was simulated employing mesoscale MD simulations, by use of single site representation of water and oil. ${ }^{8}$ Chen et al. used a similar method to investigate the structural properties of the microemulsion droplets. ${ }^{9}$ The density profile of the microemulsion beads have also been given in an oil/water/CTAB system. ${ }^{10}$ More recent works have focused on the structure and flexibility of interfacial surface in Aerosol OT (docusate sodium) micelles. ${ }^{11,12}$

Our previous work detailed coarse grained MD simulation of a micelle of polysorbate 80 along with a self-assembly study of the micelle. ${ }^{13}$ In brief, the report detailed dynamics and structural properties of the micelle as well as a single polysorbate 80 molecule. In this work, we tend to extend our work to a microemulsion system containing glyceryl trioctanoate as oil phase, ethanol as co-surfactant, and polysorbate 80 as surfactant in water, a system which has been experimentally investigated too. ${ }^{14}$ Since the ingredients that have been used in this work are pharmaceutically approved, this microemulsion has the potential to be used in various delivery routes. We aim to investigate the possible structural changes occurred by introducing the oil molecules into the micelle. To do so, a micellar system with and without co-surfactant was compared with the microemulsion droplet. To avoid enlarging, detailing some parameters such as diffusion constant, which are not directly related to structural changes, have been excluded from this article. The obtained data provides insight into the structural differences between a micelle and a microemulsion droplet and may be considered as a complementary work to our previous experiments. ${ }^{15,16}$

\section{Methodology}

\section{Molecular Model}

The molecular model of the polysorbate 80 molecule was considered, as detailed previously, ${ }^{13}$ with modifications adopted from mapping polyethylene glycol. ${ }^{17}$ Briefly, five bead types were employed including $-\mathrm{CH}_{n}-\mathrm{CH}_{2}-\mathrm{CH}_{2}-\mathrm{CH}_{2}-,-\mathrm{CH}_{n}-\mathrm{CH}_{2}-$ $\mathrm{CH}=\mathrm{CH}-,-\mathrm{CH}_{2}-\mathrm{CO}-\mathrm{O}-,-\mathrm{CH}_{n}-\mathrm{CH}_{n}-\mathrm{O}_{-}$, and $-\mathrm{CH}_{n}-\mathrm{CH}_{n}-$ $\mathrm{OH}$ for the surfactant molecule. The oil molecule consisted of

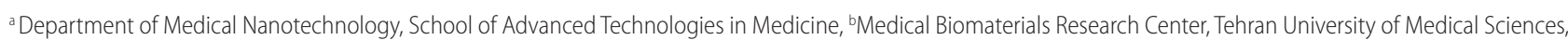
Tehran, Iran

'Department of Computer Science, School of Mathematics, Statistics, and Computer Sciences, University of Tehran, Tehran, Iran.

Correspondence to Amir Amani (email: aamani@sina.tums.ac.ir).

(Submitted: 29 September 2014 - Revised version received: 27 December 2014 - Accepted: 02 January 2015 - Published online: Winter 2015)
} 
$-\mathrm{CH}_{n}-\mathrm{CH}_{2}-\mathrm{CH}_{2}-\mathrm{CH}_{2}-$ and $-\mathrm{CH}_{2}-\mathrm{CO}-$ $\mathrm{O}-$ bead types and ethanol was taken as a single bead. The parameters of bonded and non-bonded interactions were taken from previous reports of MARTINI force field. ${ }^{18,19}$ MD simulations were performed by DLPOLY_2.1920 in the NPT ensemble, with pressure and temperature set at 1 bar and $310^{\circ} \mathrm{K}$, respectively. The Berendsen's algorithm was employed with barostat and thermostat relaxation times fixed at 1.0 ps. The van der Waals interactions had a cutoff value of $12.0 \AA$ and the timestep was $40 \mathrm{fs}$.

Our previous experimental results have shown that in the most stable form, the intended microemulsion system contains 170 molecules of ethanol and 17 oil molecules per 60 molecules of polysorbate $80 .{ }^{14}$ The phase diagram of the microemulsion has also been given previously. ${ }^{16}$ To study the structural changes when moving from a micelle to a microemulsion (i.e. from having surfactant only molecules in water to having a mixture of surfactant, co-surfactant and oil molecules, dispersed in water) the following four systems were simulated:

1. a single micelle system which was previously self assembled ${ }^{13}$ with 60 surfactant molecules (i.e. aggregation number of polysorbate $80^{21}$ in 10323 water beads (MI),

2. a micelle plus co-surfactant system (i.e. random replacement of 170 water beads in MI with ethanol to yield MI-A),

3. a microemulsion system containing 60 surfactant molecules, 170 co-surfactants, and 8 oil molecules (i.e. having half the oil molecules of the ultimate microemulsion, ME-0.5), and

4. a microemulsion with 60 surfactant molecules, 170 co-surfactants, and 17 oil molecules to represent a whole droplet of microemulsion (ME-whole).

In other words, MI, MI-A, ME-0.5, and ME-whole represent systems containing surfactant/water, surfactant/co-surfactant/water, surfactant/co-surfactant/ oil/water, and surfactant/co-surfactant/ oil/water, respectively. In ME- 0.5 and ME-whole, oil molecules were initially positioned randomly.

To obtain the true simulation time, the overall time was scaled by 4 , as suggested by the MARTINI force field. The simulations were performed for $100.0 \mathrm{~ns}$, of which the first 5.0 ns was taken as equilibration and the remaining was used to extract the averages. During the equilibration period, parameters such as potential energy and eccentricity of formed droplets were monitored to confirm reaching the equilibrium, beyond which no substantial change is observed in these parameters.

\section{Results and Discussion}

Figures 1 and 2 illustrate variation of potential energy of the system as well as eccentricity of the assembled droplets during simulation period. As the details show, a fall in the potential energy is observed during the first $\sim 1.0 \mathrm{~ns}$, of which the first half is associated with a sharp fall, followed by a gradual decrease in the second half. It is arguable that during the first $\sim 0.5 \mathrm{~ns}$, some selfassembly processes occur, while in the time range $0.5-1.0 \mathrm{~ns}$, only rearrangement of surfactant/co-surfactant/oil molecules happen to obtain the most stable droplet. A similar pattern is observed for the eccentricity of the formed droplets. Therefore, an approximate equilibration period of $1.0 \mathrm{~ns}$ appears to be sufficient for stabilizing the droplets.
Fig. 3 shows snapshots of the equilibrated systems under study. As shown in Fig. 3a, the micelle forms a nearly spherical shape, as reported previously. ${ }^{13}$ In addition, polar groups of the micelle are laid in a hydrophilic shell, covering the hydrophobic moieties of the surfactants to form a typical micelle droplet. In Fig. 3b, where co-surfactant is added to the micellar system, the ethanol molecules appear to distribute throughout the system with few diffusing to the droplet. These molecules remain in the hydrophilic parts of the surfactant molecule and may contribute to form a shell around the hydrophobic core. Oil molecules then move to the centre of the droplets to form the oil-inwater microemulsion droplets and lie between the surfactant hydrophobic and hydrophilic chains (see Fig. 3c, d). These findings are verified by the details given in Fig. 4 and Tables 1 and 2, as will be discussed later.

The radii of gyration $\left(R_{g}\right)$ of the whole droplets as well as the hydrophobic core, consisting of hydrophobic tails of the surfactant and the oil molecules, for the four systems under study have been given in Table 1. From the results, by introducing oil molecules, no

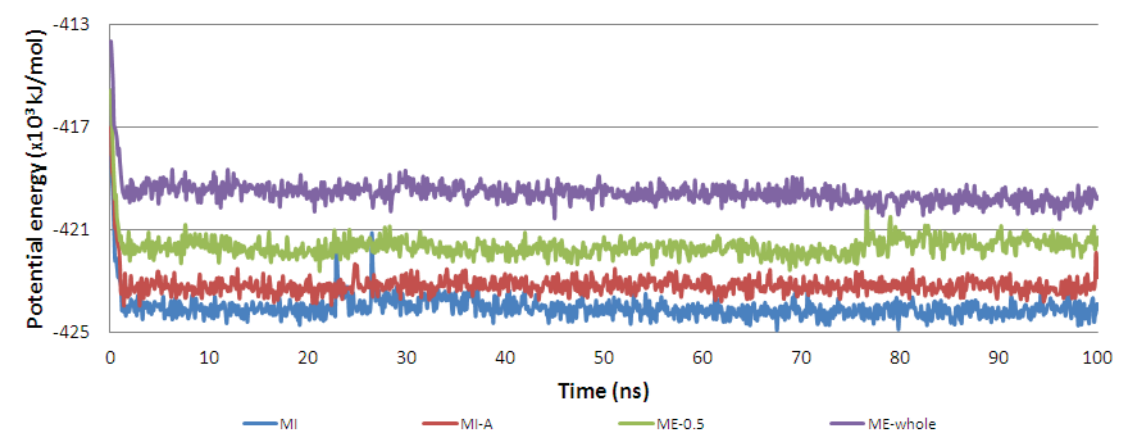

Fig. 1 Change in potential energy of the systems during simulation time.

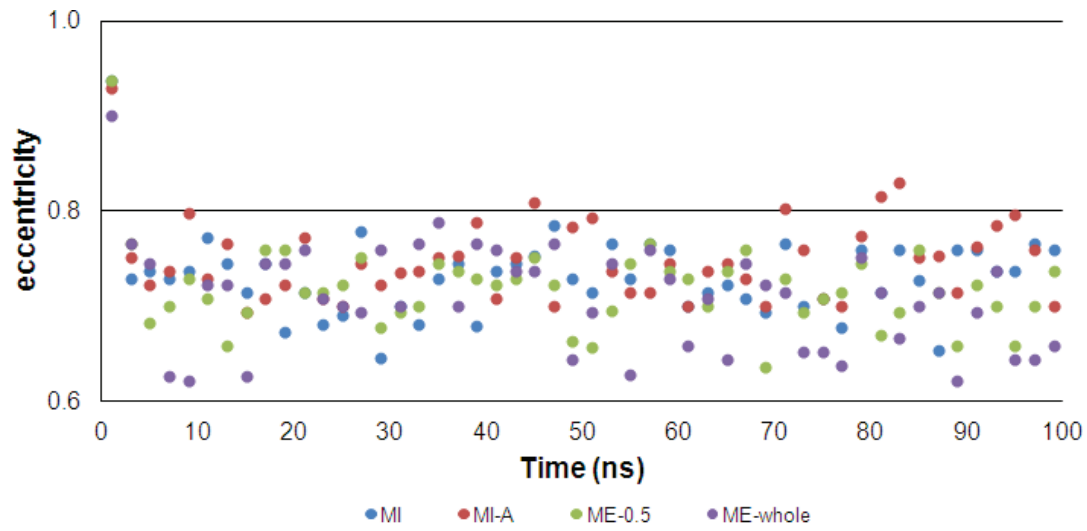

Fig. 2 Eccentricity of four systems as a function of simulation time. 

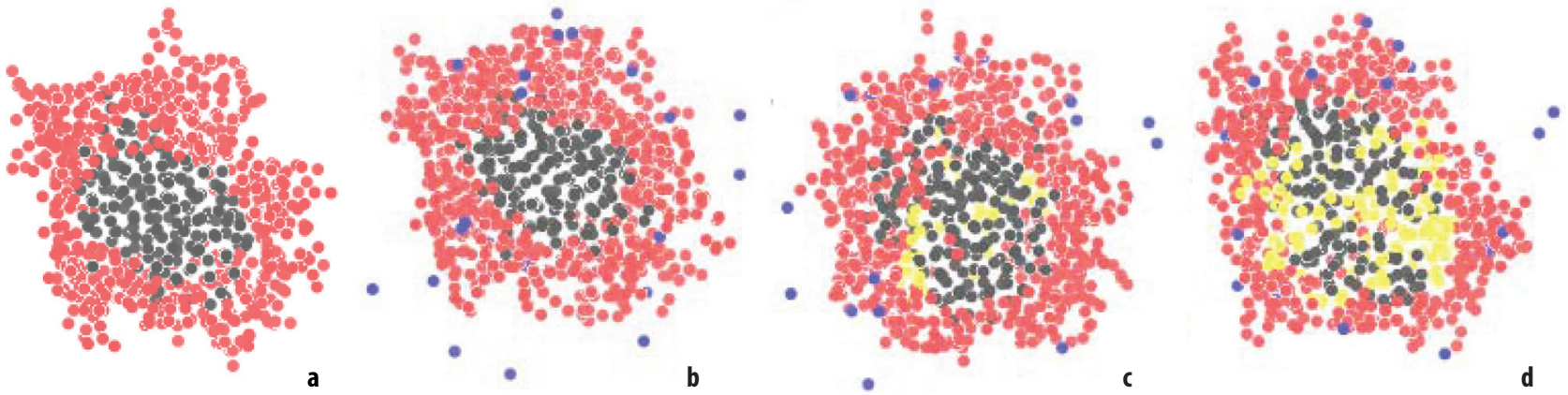

Fig. 3 Snapshots of the equilibrated droplets for the MI (a), MI-A (b), ME-0.5 (c), and ME-whole (d). The hydrophilic, hydrophobic, ethanol, and oil moieties are represented by red, black, blue, and yellow colours, respectively. A core-shell system has been formed in all the system, having oil and hydrophobic parts of surfactant in the core and hydrophilic parts in the shell, wherever applicable.
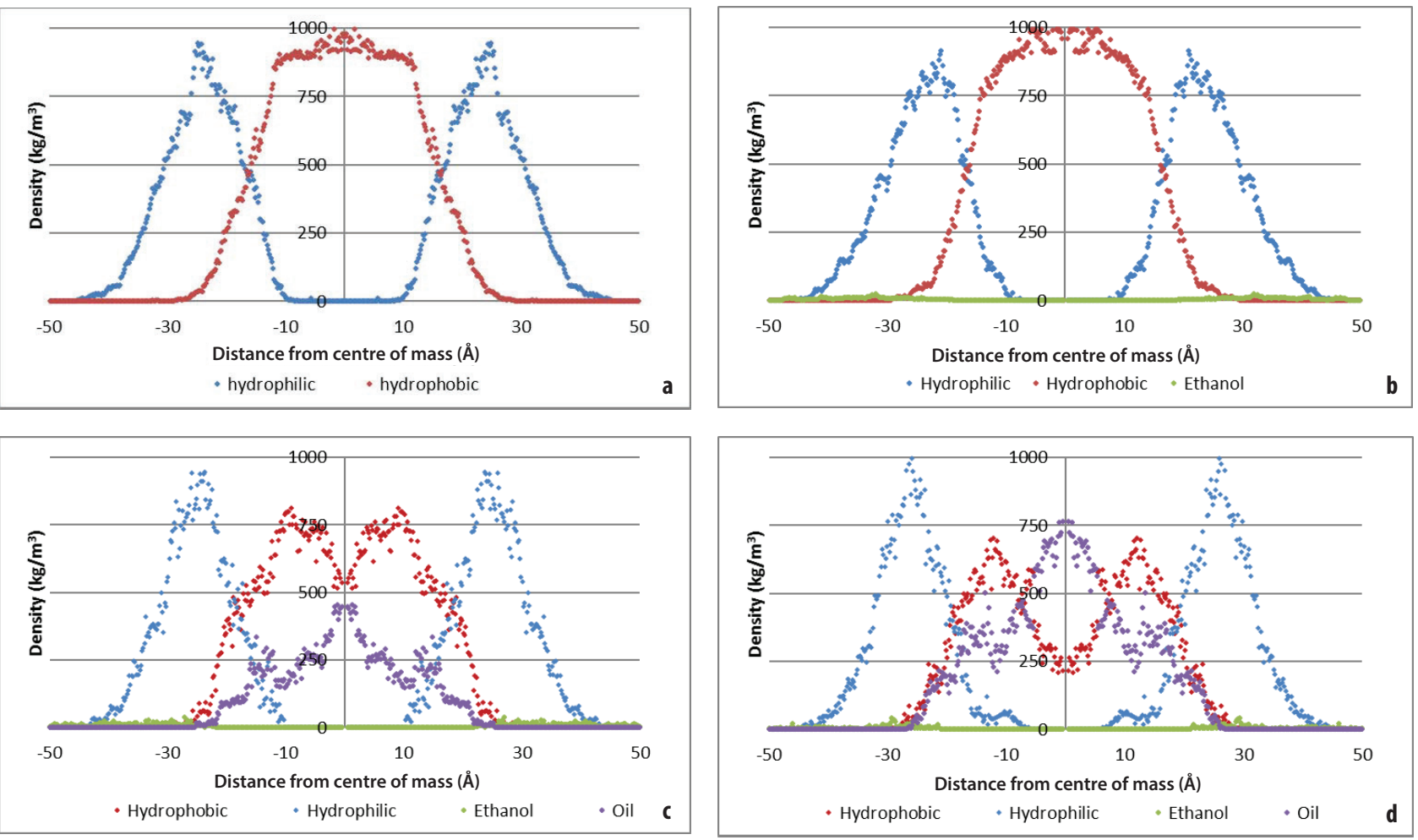

Fig. 4 Density profile of different systems as regards with the droplets centre of mass (a) MI, (b) MI-A, (c) ME-0.5, and (d) ME-whole. The centre of droplets mainly consisted of oil, covered by hydrophobic parts of the surfactant. The shell mostly contains the hydrophilic parts of the surfactant and the co-surfactant, wherever applicable.

\section{Table 1. Radius of gyration of the whole droplet as well as the hydrophobic core in the systems under study, averaged over the trajectory}

\begin{tabular}{lcc}
\hline & Whole $\operatorname{droplet}(\mathbf{A})$ & Hydrophobic core $(\AA)$ \\
\hline MI & 26.32 & 16.00 \\
MI-A & 26.55 & 15.87 \\
ME-0.5 & 26.45 & 16.58 \\
ME-whole & 26.59 & 17.74 \\
\hline
\end{tabular}

ME: microemulsion; Ml: micelle.

\section{Table 2. The obtained ellipsoidal semiaxes (a, b, and c) and the eccentricity (e) of the systems under study}

\begin{tabular}{lccccc}
\hline & $\mathbf{a}(\AA)$ & $\mathbf{b}(\AA)$ & $\mathbf{c}(\AA)$ & $<\mathbf{a} / \mathbf{c}\rangle$ & $\mathbf{e}$ \\
\hline MI & 38.65 & 35.93 & 26.08 & 1.48 & 0.73 \\
MI-A & 39.22 & 36.25 & 25.92 & 1.51 & 0.75 \\
ME-0.5 & 39.04 & 34.88 & 27.52 & 1.42 & 0.71 \\
ME-whole & 38.74 & 35.65 & 27.62 & 1.40 & 0.70 \\
\hline
\end{tabular}

ME: microemulsion; MI: micelle. important change in the size of the whole droplet is observed (26.59 $\AA$ vs. $26.32 \AA)$. This is not consistent with the general understanding about microemulsions as they are commonly known as swollen micelles. ${ }^{22}$ This is probably due to fact that in a typical microemulsion, concentrations of oil and surfactant are more or less similar. Thus, a considerable volume of nanoemulsion droplet is expected to be occupied by oil molecules. However, in the system understudy, the total number of oil beads is 153 which is substantially smaller than the number of surfactant beads - 1680 . 
On the other hand, oil molecules have penetrated into the hydrophobic core and enlarged it (i.e. from $16.00 \AA$ to $17.74 \AA$ ). . The effect of this increase in the core size on the size of whole droplet is neutralized by stretching (i.e. thinning) the hydrophilic shell, thus, only a small change (i.e. $<0.3 \AA$ ) is observed in the whole droplet size. The lack of a considerable change in the radius of gyration of micelle (MI) compared with microemulsion (ME-whole) has been experimentally reported in a similar system. ${ }^{14}$

Additionally, comparing the $R_{g}$ values of MI with MI-A in Table $\stackrel{g}{1}$ shows that when ethanol molecules are introduced to the system, a small decrease in radius of gyration of the core is observed while the whole droplets becomes somewhat larger. This is due to fact that some of the co-surfactant molecules go into the hydrophilic part of the micelle (i.e. micelle shell) which leads to expanding the shell. Simultaneously, the ethanol molecules "push" the hydrophobic parts of the surfactants together, making a degree of packing the core part of the micelle.

Using the equation $R=\sqrt{5 / 3} R_{g}$, the radius of the droplets in each system may be estimated as 33.98, $34.28,34.15$, and $34.33 \AA$ for MI, MI-A, NE-.5, and ME-whole, respectively. The deviation of the radius and $R_{g}$ of the micelle in current work from that of our previous report (i.e. $27.55 \AA$ ) is most probably due to the modifications performed on the mapping polyethylene glycol and alkyl parts of the surfactant.

To characterize the droplets shape, an ellipsoid was assumed for each droplet, having three semiaxes, $a, b$, and $c$. The semiaxes were computed using principal axes of inertia in the droplet $I_{1}$ $>I_{2}>I_{3}$ by:

$$
\begin{aligned}
& I_{1}=\frac{1}{5} M\left(a^{2}+b^{2}\right) \\
& I_{2}=\frac{1}{5} M\left(a^{2}+c^{2}\right) \\
& I_{3}=\frac{1}{5} M\left(b^{2}+c^{2}\right)
\end{aligned}
$$

where total mass of droplet is shown by $M$ and the eccentricity of the droplets was computed by the following equation:

$$
e=\sqrt{1-\frac{c^{2}}{a^{2}}}
$$

Table 2 lists the shape parameters of the systems. Considering the details in Table 2 , which lists the shape parameters of the droplets, a prolate ellipsoid is obtained for all the systems. Similar shapes have been documented for micelles with different surfactant types. ${ }^{13,23,24}$ Furthermore, comparing MI with MI-A systems, a slight increase in the eccentricity is observed. This shows that the ethanol molecules which have entered the hydrophilic shell have made the droplets more elongated. While, addition of oil molecules has made the droplet slightly more spherical (i.e. less eccentricity and $\mathrm{a} / \mathrm{c}$ values). To investigate this effect, one may consider the packing parameter, $P$, as a useful approach in predicting the geometry of micelles:

$$
P=\frac{V}{a_{0} l_{c}}
$$

where $V, a_{0}$, and $l_{c}$ represent the volume of hydrophobic tail, the headgroup area, and the maximum length of the tail. ${ }^{25}$ Spherical micelles have $\mathrm{P}$ values $<1 / 3$, while non-spherical ones are characterized by higher values for P. In our previous work, a packing parameter of $\sim 0.12$ was determined for the surfactants in the micelle. ${ }^{13}$ It is arguable that when oil molecules are introduced to the system, they enter the hydrophobic core and squeeze the hydrophobic tails of the surfactants. Therefore, the volume occupied by the hydrophobic tails decreases (i.e. $V$ ) and the packing parameter decreases too. Consequently, a more spherical micelle may be expected from the system. Similarly, ethanol molecules contribute to making smaller values for the headgroup area and larger values for P. As a result, deviation from sphere could be expected for the droplet. Undoubtedly, such changes in the micelle shape may be associated with changes in the thermodynamic stability of the system ${ }^{26}$ and further studies are required to examine it.

Fig. 2 shows the average density profiles of the systems. As described previously, ${ }^{13}$ the effect of the deviation of the droplets shape from sphere was ignored. In our previous work, the density profiles of the following moieties were reported for the polysorbate 80 : the hydrophobic tails, the core parts of the molecule, the terminal polar droplets having hydroxyl groups, and the hydrophilic parts. In this work, to make the comparison between the four systems easier, the molecular structure of polysorbate 80 was divided into two distinct groups: a hydrophilic part (containing $-\mathrm{CH}_{2}-\mathrm{CO}-\mathrm{O}-,-\mathrm{CH}_{n}-\mathrm{CH}_{n}-\mathrm{O}-$, and $\left.-\mathrm{CH}_{n}-\mathrm{CH}_{n}-\mathrm{OH}\right)$ and a hydrophobic part (having $-\mathrm{CH}_{n}-\mathrm{CH}_{2}-\mathrm{CH}_{2}-$ $\mathrm{CH}_{2}-$ and $-\mathrm{CH}_{n}-\mathrm{CH}_{2}-\mathrm{CH}=\mathrm{CH}-$ ). Therefore, the density distribution of hydrophobic part, hydrophilic part, oil, and ethanol were computed.

From Fig. 4a, the core of the micelle consists merely of hydrophobic beads and the hydrophilic particles come into sight at $\sim 8 \AA$ from the centre of mass. Comparing the data from Fig. $4 \mathrm{a}$ and $\mathrm{b}$, no considerable change in the distribution of surfactant droplets may be observed by the addition of ethanol molecules. The closest ethanol molecules to the centre of mass lie $\sim 18 \AA$ from centre. Their density then starts to increase to some extent and a peak in concentration appears to be $\sim 32 \AA$ off centre. This peak could be due to interactions between the ethanol molecules and the hydrophilic parts of the polysorbate 80 , especially the beads having hydroxyl groups. The interaction level for these beads with ethanol is defined as level II in the forcefield, ${ }^{19}$ showing strong attractions between them, probably due to the existence of hydrogen bonds. Remembering the important effect of co-surfactants in forming microemulsion droplets, this peak could be associated with forming stable microemulsion droplets.

Moving on to the Fig. $4 \mathrm{c}$ and d, the oil molecules appear to push the hydrophobic parts and locate into the centre of the droplet. Their density then starts to decrease and the oil molecules disappear at $\sim 25 \AA$ and $\sim 27 \AA$ from centre of mass for the ME-0.5 and ME-whole, respectively. Interestingly, at 12-15 from centre, a transient increase in the density of oil is observed, at which the density of oil and hydrophilic parts of the polysorbate 80 are more or less equal. Considering the two bead types present in the oil, the alkyl group (i.e. $-\mathrm{CH}_{n}-\mathrm{CH}_{2}-\mathrm{CH}_{2}-\mathrm{CH}_{2}-$ ) may not be the cause as the group has a strong hydrophobic nature, thus, cannot make suitable interactions with the hydrophilic parts of the surfactant. We believe that the increase in concentration of oil is due to its $-\mathrm{CH}_{2}-\mathrm{CO}-\mathrm{O}-$ bead types which could make suitable interactions with the hydrophilic parts of the surfactant (i.e. interaction level IV). 


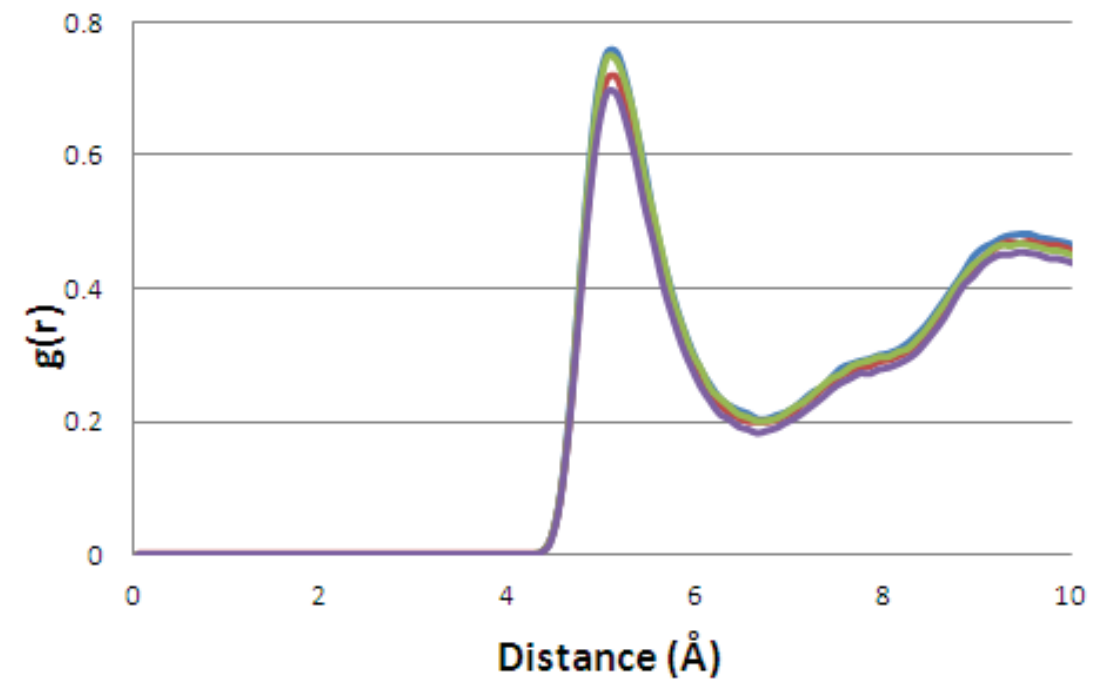

MI $-\mathrm{MI}-\mathrm{A} \longrightarrow \mathrm{ME}-0.5 \longrightarrow$ ME-whole

Fig. 5 Averaged radial distribution functions for the four systems with water beads.

Furthermore, by adding oil to the system, the ethanol molecules are driven from the droplet: they start to show up at $\sim 21 \AA$ from centre of mass in ME-0.5 and ME-whole, compared with $\sim 18 \AA \AA$ in MI-A.
Moreover, the radial distribution function, $g(r)$, of each of the moieties was computed and reported in Fig. 5. As the details show, the hydration shells do not appear to be different in the four systems, showing the importance of the shells, which are composed of surfactant molecules, in interaction with water molecules.

\section{Conclusion}

The MD simulations of a nano-system containing polysorbate 80 as surfactant with or without ethanol (i.e. co-surfactant) and oil were performed in this study to identify the possible structural changes. In general, oil molecules tend to move to the central region of the droplets, thus, making the hydrophobic core of the droplets larger. Interestingly, insertion of oil/ethanol does not influence the overall size substantially. It was also found that addition of oil molecules make the droplets more spherical, while ethanol molecules deviate the aggregate from a spherical shape.

\section{Acknowledgment}

The study has been funded by Tehran University of Medical Sciences grant number 88-01-87-8624.

\section{References}

1. Danielsson I, Lindman B. The definition of microemulsion. Colloids Surf 1981 Dec;3(4):391-2. doi: http://dx.doi.org/10.1016/0166-6622(81)80064-9

2. Lawrence MJ, Rees GD. Microemulsion-based media as novel drug delivery systems. Adv Drug Deliv Rev. 2000 Dec 6;45(1):89-121. doi: http://dx.doi. org/10.1016/s0169-409x(00)00103-4 PMID: 11104900

3. Tenjarla S. Microemulsions: an overview and pharmaceutical applications. Crit Rev Ther Drug Carrier Syst. 1999;16(5):461-521. doi http://dx.doi. org/10.1615/critrevtherdrugcarriersyst.v16.i5.20 PMID: 10635455

4. Aulton ME. Aulton's pharmaceutics: the design and manufacture of medicines. Edinburgh: Churchill Livingstone; 2007.

5. Bagwe RP, Kanicky JR, Palla BJ, Patanjali PK, Shah DO. Improved drug delivery using microemulsions: rationale, recent progress, and new horizons. Crit Rev Ther Drug Carrier Syst. 2001;18(1):77-140. doi: http:// dx.doi.org/10.1615/critrevtherdrugcarriersyst.v18.i1.20

6. Baaden M, Burgard M, Wipff G. TBP at the water-oil interface: the effect of TBP concentration and water acidity investigated by molecular dynamics simulations. J Phys Chem B. 2001 Nov;105(45):11131-41. doi: http://dx.doi. org/10.1021/jp011890n

7. Baaden M, Schurhammer R, Wipff G. Molecular dynamics study of the uranyl extraction by tri-n-butylphosphate (TBP): demixing of water/"oil"/ TBP solutions with a comparison of supercritical $\mathrm{CO}_{2}$ and chloroform. J Phys Chem B. 2002 Jan;106(2):434-41. doi: http://dx.doi.org/10.1021/jp0123785

8. Bearchell CA, Heyes DM. Mesoscale modelling studies of microemulsions. Phys Chem Chem Phys. 2001 Nov 26;3(23):5255-65. doi: http://dx.doi. org/10.1039/b105096f

9. Chen Z, Cheng X, Cui H, Cheng P, Wang H. Dissipative particle dynamics simulation of the phase behavior and microstructure of CTAB/octane/1butanol/water microemulsion. Colloids Surf A Physicochem Eng Asp. 2007 Jul;301(1-2):437-43. doi: http://dx.doi.org/10.1016/j.colsurfa.2007.01.022

10. Li Y, Guo Y, Bao M, Gao X. Investigation of interfacial and structural properties of CTAB at the oil/water interface using dissipative particle dynamics simulations. J Colloid Interface Sci. 2011 Sept;361 (2):573-80. doi: http://dx.doi.org/10.1016/j.jcis.2011.05.078

11. Pieniazek PA, Lin Y-S, Chowdhary J, Ladanyi BM, Skinner JL. Vibrational spectroscopy and dynamics of water confined inside reverse micelles. J Phys Chem B. 2009 Nov 12;113(45):15017-28. doi: http://dx.doi. org/10.1021/jp906784t
12. Nevidimov AV, Razumov VF. Molecular dynamics simulations of AOT reverse micelles' self-assembly. Mol Phys. 2009 Oct 20;107(20):2169-80. doi: http:// dx.doi.org/10.1080/00268970903203736

13. Amani A, York P, de Waard H, Anwar J. Molecular dynamics simulation of a polysorbate 80 micelle in water. Soft Matter. 2011;7(6):2900-8. doi: http:// dx.doi.org/10.1039/c0sm00965b

14. Amani A. Design, characterisation and in vitro aerosolisation performance of nanoemulsion for nebulisation of hydrophobic drugs (PhD thesis). University of Bradford; 2008.

15. Amani A, York P, Chrystyn H, Clark BJ. Factors affecting the stability of nanoemulsions - use of artificial neural networks. Pharm Res. 2010 Jan;27(1):37-45. doi: http://dx.doi.org/10.1007/s1 1095-009-0004-2 PMID: 19908130

16. Amani A, York P, Chrystyn H, Clark BJ, Do DQ. Determination of factors controlling the particle size in nanoemulsions using artificial neural networks. Eur J Pharm Sci. 2008 Sep 2;35(1-2):42-51. doi: http://dx.doi. org/10.1016/j.ejps.2008.06.002 PMID: 18617002

17. Lee $H$, de Vries AH, Marrink SJ, Pastor RW. A coarse-grained model for polyethylene oxide and polyethylene glycol: conformation and hydrodynamics. J Phys Chem B. 2009 Oct 8;113(40):13186-94. doi: http://dx.doi.org/10.1021/jp9058966 PMID: 19754083

18. Lee H, Pastor RW. Coarse-grained model for PEGylated lipids: effect of PEGylation on the size and shape of self-assembled structures. J Phys Chem B. 2011 Jun 23;15(24):7830-7. doi: http://dx.doi.org/10.1021/jp2020148 PMID: 21618987

19. Marrink SJ, Risselada HJ, Yefimov S, Tieleman DP, De Vries AH. The MARTINI force field: coarse grained model for biomolecular simulations. J Phys Chem B. 2007 Jul 12;111(27):7812-24. doi: http://dx.doi.org/10.1021/ jp071097fPMID: 17569554

20. ForesterTR, Smith W. DL_POLY_2.0-a general-purpose parallel molecular dynamics package. J Mol Graph. 1996 Jun;14(3):136-41. doi: http://dx.doi. org/10.1016/s0263-7855(96)00043-4 PMID: 8901641

21. de Campo, L, Yaghmur A, Garti N, Leser ME, Folmer B, Glatter O. Fivecomponent food-grade microemulsions: structural characterization by SANS. J Colloid Interface Sci. 2004 Jun 1;274(1):251-67. doi: http://dx.doi. org/10.1016/j.jcis.2004.02.027 PMID: 15120300 
22. Zulauf M, Eicke HF. Inverted micelles and microemulsions in the ternary system water/aerosol-OT/isooctane as studied by photon correlation spectroscopy. J Phys Chem. 1979 Feb;83(4):480-6. doi: http://dx.doi. org/10.1021/j100467a011

23. Abel S, Sterpone F, Bandyopadhyay S, Marchi M. Molecular modeling and simulations of AOT-water reverse micelles in isooctane: structural and dynamic properties. J Phys Chem B. 2004 Dec;108(50):19458-466. doi: http://dx.doi.org/10.1021/jp047138e
24. Senapati S, Keiper JS, DeSimone JM, Wignall GD, Melnichenko YB, Frielinghaus $\mathrm{H}$, Berkowitz ML. Structure of phosphate fluorosurfactant based reverse micelles in supercritical carbon dioxide. Langmuir. 2002 Oct:18(20):7371-6. doi: http://dx.doi.org/10.1021/la025952s

25. Tanford C. Micelle shape and size. J Phys Chem. 1972 Oct;76(21):3020-4. doi: http://dx.doi.org/10.1021/j100665a018

26. Leitmannova Liu A. Advances in planar lipid bilayers and liposomes. London: Academic Press; 2011 